\title{
Analytical and sensory data correlation to understand consumers' grape preference
}

\author{
T. Basile, R. Perniola, M.F. Cardone, A.D. Marsico, and D. Antonacci
}

Consiglio per la ricerca in agricoltura e l'analisi dell'economia agraria -Centro di ricerca Viticoltura ed Enologia (CREA- VE), Via Casamassima 148, 70010 Turi (Ba), Italy

\begin{abstract}
NIR spectroscopy is a rapid, economic and not destructive technique employed in food analysis. Concerning fresh table grape, the analysis is usually limited to juices, homogenates or skin extracts which usually give better NIR prediction models. Scanning of intact berries is challenging since each berry has specific features (berry shape, presence of superficial pigmentation, etc.) and, moreover, there are punctual variations even within the same berry. It would be of great interest to obtain information about maturity parameters and consumer's appreciation directly from intact berries, since it would save both time and money. In this article, near infrared (NIR) spectroscopy and chemometric methods have been employed to search for a correlation between sensory analysis and analytical data. The research findings show how it is possible to use a rapid, economic and not destructive emerging technology such as NIR spectroscopy to understand consumer's preference directly from intact berries.
\end{abstract}

\section{Abstract}

Chromatographic (HPLC), spectrometric (NIR) and enzymatic methods together with sensory analysis have been employed in a two-year survey on grape varieties harvested from the experimental vineyard of CREA Research Centre for Viticulture and Enology of Turi together with samples obtained from Italian companies leading producers of table grape. The results allowed to enlighten the positive acceptance of grapes through a correlation between sensory analysis and analytical results. It is well known that the organoleptic properties of table grape greatly depend on the content and composition of sugars and organic acids since the balance between sweetness and acidity is a basic precept in men's judgment of the quality of many fruits. The acidity is another character of prime sensory importance for grape flavour. The principal organic acids found in grapes are tartaric, malic and to a small extent, citric. Each acid has its own sensory attributes; thus, all individual organic acids play a different role in defining the organoleptic properties of the grape. Not only sugars and organic acids but also some phenolic and aromatic compounds contribute to influence the perceived grape flavour. Moreover, human perceptions of food and beverages are the results of complex sensory and interpretation processes in which there is a contribution of a combination of visual, olfactory, taste and texture inputs as well as cognitive factors. The perception of such multidimensional stimuli as conducted by human nervous system are difficult or impossible to predict from instrumental measures. Indeed, sensory analysis is often employed in the evaluation of consumers' appreciation of table grape. The correlation of sensory characterization and the analytical techniques commonly employed in the grape sector hardly provides a complete explanation of sensory preference. The NIR spectroscopy allows various food matrices to be analysed with little or no sample preparation in a simple, economical, fast and non-destructive way. The results of this work show how NIR spectroscopy seems not only an interesting technique able to predict some important grape quality parameters even from intact berries, but could be used to correlate consumers' preference with analytical data obtained with analysis techniques commonly used in table grape sector (e.g. HPLC).

\section{Material and methods}

The sugar content, conventionally expressed as total soluble solids (TSS) and the titratable acidity (TA) are the main parameters for fresh Vitis vinifera $\mathrm{L}$ table grape quality evaluation. Indeed, TSS and TA values are the only parameters which have to satisfy a specific minimal value for grape commercialization [1-3]. An initial screening on table grape varieties grown in experimental vineyards of CREA-VE of Turi (Azienda Sperimentale "Lamarossa", Rutigliano, Bari, Southern Italy Lat. 40,956 N, Lon. 17,008 E) based on consumer acceptability from experiments involving hedonistic testing taste trials was performed in 2017. The sensorial analysis showed how for some varieties TSS below $12.5^{\circ}$ Brix, and a TSS/TA ratio lower than the recommended 20/1 (thus not commercializable), the consumers acceptability was greater compared to other varieties with ${ }^{\circ}$ Brix and TSS/TA values within the maturity requirements [4]. Therefore, we decided to perform a sensory evaluation on grape samples with different maturity levels, even below the commercialization ones. In order to understand if the reasons behind grape appreciation were different for grapes with different features (white or red, seeded or seedless), among the grape varieties already tested three varieties with different features have been employed: a 
Table 1. Maturity parameters and sensory data.

\begin{tabular}{|c|c|c|c|c|c|}
\hline & \multicolumn{3}{|c|}{ Maturity parameters $\dagger$} & \multicolumn{2}{c|}{ Sensory data $\neq$} \\
\hline Variety & ${ }^{\circ}$ Brix & TA & TSS/TA & \%Acceptance & Preference \\
\hline \multirow{4}{*}{ Melissa } & $20.3 \pm 0.1$ & $3.0 \pm 0.1$ & 67 & 79 & $6.30 \pm 1.21^{\mathrm{a}}$ \\
\cline { 2 - 6 } & $19.1 \pm 0.1$ & $3.8 \pm 0.0$ & 50 & 65 & $6.00 \pm 0.63^{\mathrm{a}}$ \\
\cline { 2 - 6 } & $18.3 \pm 0.1$ & $6.0 \pm 0.1$ & 31 & 40 & $7.00 \pm 1.67^{\mathrm{a}}$ \\
\cline { 2 - 6 } & $12.0 \pm 0.1$ & $10.0 \pm 0.1$ & 12 & 2 & $3.00 \pm 0.51^{\mathrm{b}}$ \\
\cline { 2 - 6 } Italia & ${ }^{\circ}$ Brix & TA & TSS/TA & \%Acceptance & Preference \\
\hline & $18.8 \pm 0.1$ & $5.0 \pm 0.1$ & 38 & 100 & $7.50 \pm 0.87^{\mathrm{a}}$ \\
\cline { 2 - 6 } & $16.8 \pm 0.1$ & $6.0 \pm 0.1$ & 28 & 83 & $6.40 \pm 1.21^{\text {ab }}$ \\
\cline { 2 - 6 } & $15.3 \pm 0.2$ & $5.9 \pm 0.4$ & 26 & 60 & $5.20 \pm 0.50^{\text {bc }}$ \\
\cline { 2 - 6 } & $12.1 \pm 0.1$ & $8.3 \pm 0.2$ & 15 & 32 & $4.30 \pm 0.50^{\mathrm{c}}$ \\
\cline { 2 - 6 } & ${ }^{\circ} \mathbf{B r i x}$ & $\mathbf{T A}$ & TSS/TA & \%Acceptance & Preference \\
\hline \multirow{5}{*}{ Red Globe } & $21.3 \pm 0.1$ & $3.5 \pm 0.1$ & 61 & 93 & $6.70 \pm 0.67^{\mathrm{a}}$ \\
\cline { 2 - 6 } & $19.0 \pm 0.1$ & $4.5 \pm 0.1$ & 42 & 100 & $6.83 \pm 0.29^{\mathrm{a}}$ \\
\cline { 2 - 6 } & $15.7 \pm 0.2$ & $4.3 \pm 0.1$ & 37 & 90 & $6.30 \pm 0.30^{\mathrm{a}}$ \\
\cline { 2 - 6 } & $14.1 \pm 0.0$ & $6.0 \pm 0.0$ & 24 & 95 & $6.10 \pm 0.40^{\mathrm{a}}$ \\
\cline { 2 - 6 } & $12.4 \pm 0.1$ & $6.9 \pm 0.0$ & 18 & 0 & $4.50 \pm 0.71^{\mathrm{b}}$ \\
\hline
\end{tabular}

Values are mean \pm DS. For each variety values in the same column bearing different letters are significantly different. Data have been analysed with $\dagger$ an ANOVA test followed by a Tukey post hoc test or $\ddagger$ the Kruskal-Wallis non-parametric test followed by the Dunn post hoc test using a $p$-value $<0.05$.

white seeded one (Italia), a white seedless one (Melissa) and a red seeded one (Red Globe). TSS ( ${ }^{\circ}$ Brix) was determined at $20^{\circ} \mathrm{C}$ using a digital refractometer Atago PR1 (Atago Co., Tokyo, Japan). TA was measured as tartaric acid $(\mathrm{g} / \mathrm{L})$ by titration of grape juice with sodium hydroxide $(0.1 \mathrm{~N})$ to an endpoint $\mathrm{pH}$ of 7 . The HPLC analysis of organic acids was performed following the Cane [5] procedure while for sugars an enzymatic assay was employed (SATURNO 150 Crony Instruments). The sensory characterisation was performed with a panel test was composed of 82 subjects (58 high school students and 24 adults). The significance of the sensory analysis was assessed performing the Kruskal-Wallis non-parametric test followed by the Dunn test as a post hoc test, using a $p$ value $<0.05$ [6]. Moreover, consumers'acceptance (\%CA) was calculated as follows: number of tasters giving a preference value over 5 divided the total number of tasters [7]. A Bruker TANGO FT-NIR spectrometer has been employed for spectra acquisition and the OPUS/QUANT software (Bruker Optik GmbH, Ettlingen Germany) Vers. 2.0 has been used for chemometric analysis. Berry samples of fresh table grape have been used to build NIR calibration models. For each berry, the NIR spectra of the intact single berry have been recorded on three different berry faces. The first derivative and the vector normalization were chosen as pre-processing techniques in accordance with their predictive performance. After cross-validation, outlier removal and optimization steps, the final version of the calibration models (PLS regression) was obtained with the OPUS/QUANT Bruker software. The $\mathrm{R}$ software environment was employed for the statistical analysis (R Core Team (2013). R: A language and environment for statistical computing. $\mathrm{R}$ Foundation for Statistical Computing, Vienna, Austria. URL http: //www.R-project.org/).

\section{Results and discussion}

\subsection{NIR prediction models}

For TA the predictability of the PLS model was not good $\left(\mathrm{R}^{2}\right.$ below 50$)$ for all the three varieties tested. Since in grape berries there is a gradient of acidity probably the penetration depth of NIR radiation did not reach the layer which contains the main acidic compounds which results in difference between the acidic actual composition and the amount of acids detected by the NIR technique. This might be the same reason of the lack of prediction ability of NIR also found for the analysis of single organic acids with HPLC. For the analysis of single sugars was employed an enzymatic assay, anyway, the prediction models built with NIR data did not give satisfactory results for individual sugars. Probably the sugar quantification obtained with the technique employed is not accurate enough for the sensitivity of the NIR analysis. Therefore, for sugars a change in the primary method employed could result in a better prediction.

\subsection{Sensory data analysis}

In Table 1 the maturity parameters and the sensory data are reported. The "preference" value expressed by testers is a complex parameter which is influenced by chemical composition (i.e. flavour) together with texture [8] and the visual appearance of berries is the first quality index for consumers appreciation. Indeed, for grapes varieties such as Melissa which has a green hue even with high glucidic content, the appreciation of consumers never reached the $100 \%$ even for very high TSS values $\left(20.3^{\circ} \mathrm{Brix}\right)$. The visual characteristics had a great influence on the consumers judgment also on grapes with a lower TSS $\left(<12.5^{\circ}\right.$ Brix $)$ an example of very low acceptance is Red Globe which is a red variety which does not reach an acceptable colouration at the beginning of the maturation process (a grape variety characteristic), indeed this grape with $12.4^{\circ}$ Brix had $0 \%$ of acceptance. On the other side, varieties with a more uniform colour, such as Italia, even at $12.1^{\circ}$ Brix showed an intermediate value of acceptance $(32 \%)$.

\subsection{NIR spectra}

Because of the high-water content, prominent NIR absorption peaks were observed around 10200, 8400, 6900 and $5600 \mathrm{~cm}^{-1}$ (Figs. 1-3) mainly due to fairly broad 


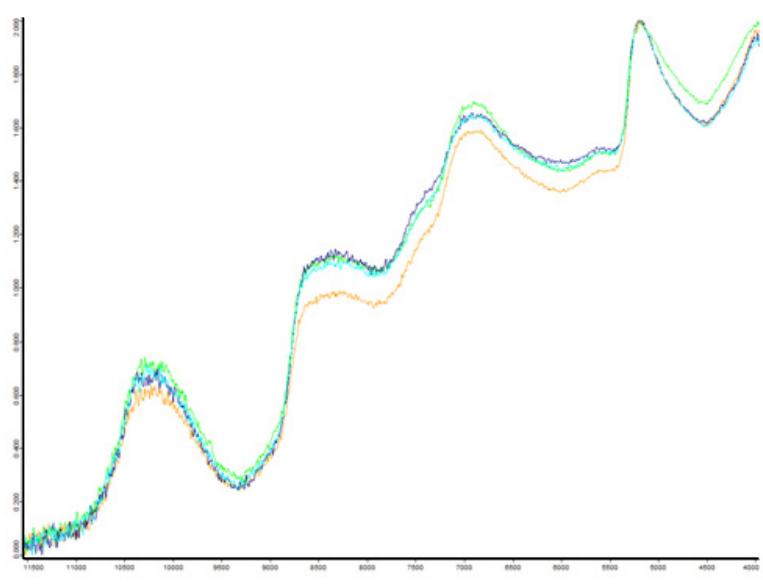

Figure 1. Averaged NIR spectra of single berries for each maturity level of Italia grape (absorbance vs. wave-numbers).

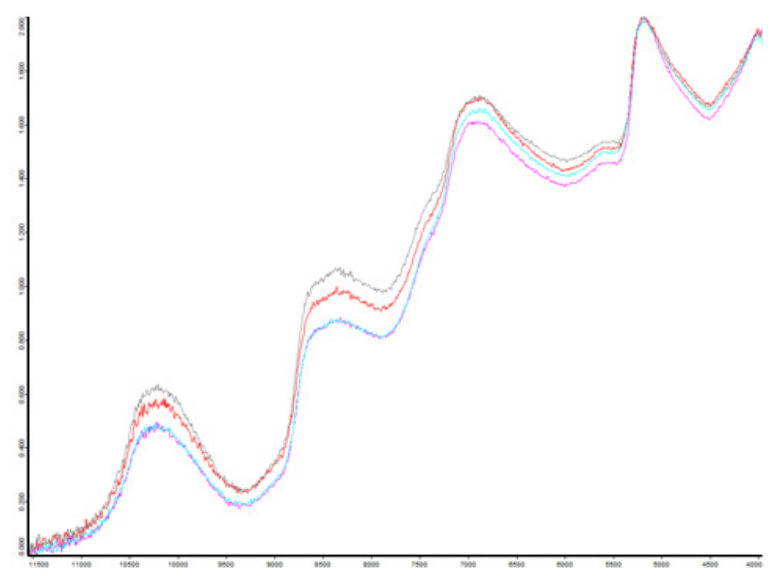

Figure 2. Averaged NIR spectra of single berries for each maturity level of Melissa grape (absorbance vs. wave-numbers).

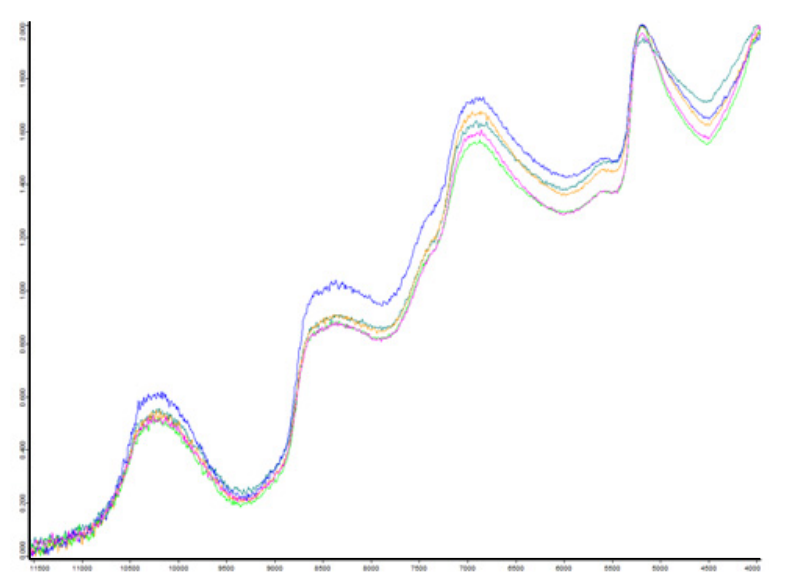

Figure 3. Averaged NIR spectra of single berries for each maturity level of Red Globe grape (absorbance vs. wavenumbers).

water absorption bands which usually dominate the NIR spectrum of fruits and vegetables [9].

Anyway, the complex hydrogen bonding interactions between water and other sample components (e.g. sugar, protein, etc.) induce a water structure variation which translates in a modification of NIR absorption bands of water $[10,11]$. These modifications are directly related to the actual sample composition thus can be employed

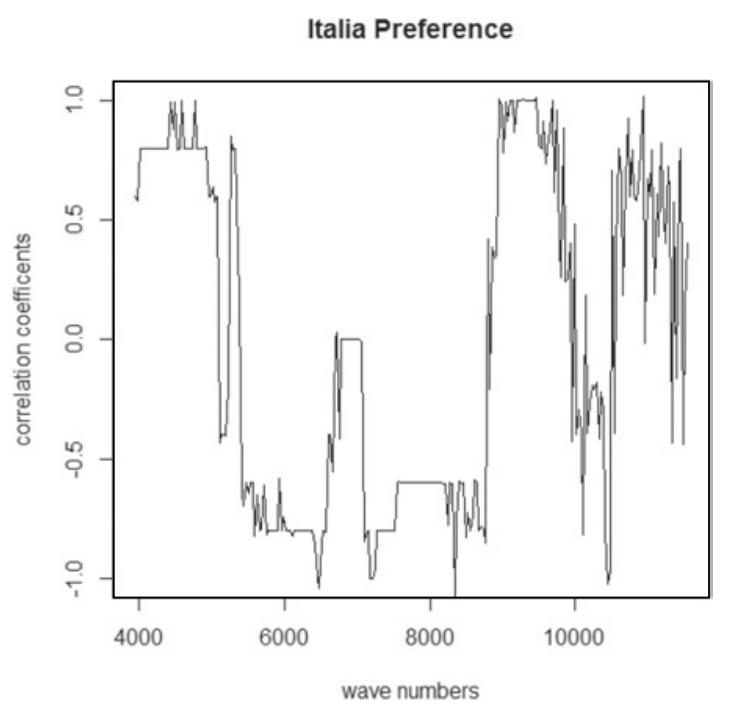

Figure 4. Correlogram: correlation coefficients between NIR spectroscopic data and the sensory data "preference" of Italia grape.

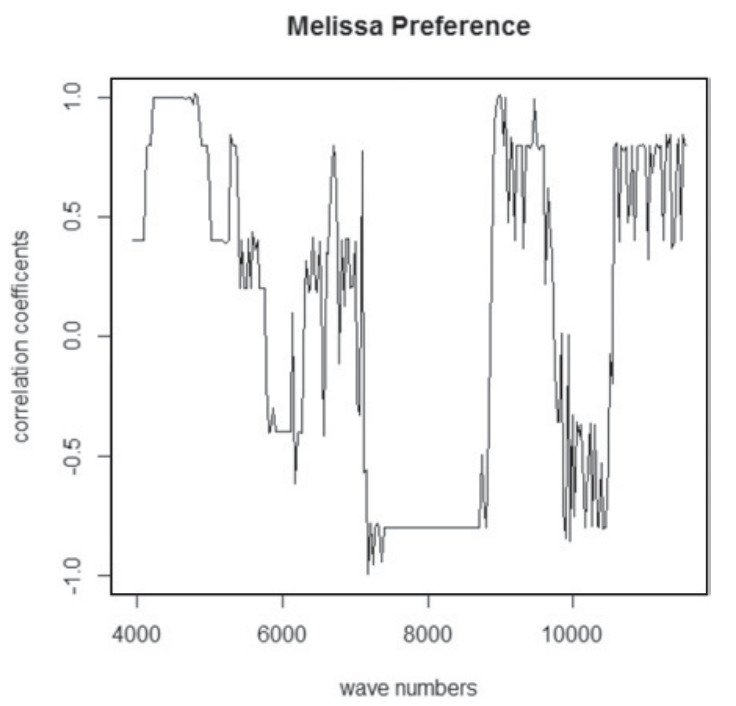

Figure 5. Correlogram: correlation coefficients between NIR spectroscopic data and the sensory data "preference" of Melissa grape.

in order to find a relation to analytical parameters (e.g. sugar content), sensory or even texture related data. Indeed, chemometrics procedures (multivariate techniques) applied to spectroscopic data are necessary employed to extract significant information from the spectra.

\subsection{NIR and sensory data}

A correlation between average sensory data and instrumental data obtained per maturity level was estimated with the non-parametric Spearman coefficients. A "correlogram" graph was plotted for the preference as a function of wavelength for each variety tested.

For the two white grape varieties the correlation between wavenumbers and preference shows a similar profile (Figs. 4-5). This led us to understand that the presence of seeds did not lead to a drastic differentiation in preference between the two white varieties. The 


\section{Red Globe Preference}

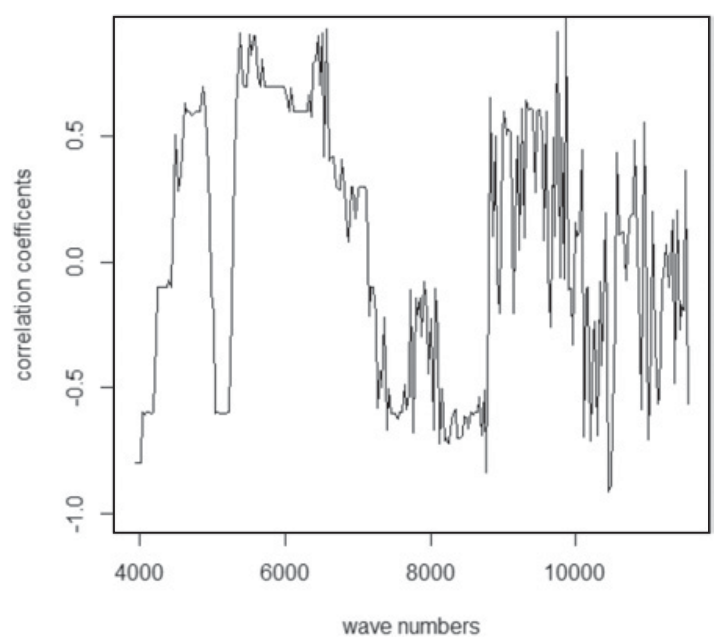

Figure 6. Correlogram: correlation coefficients between NIR spectroscopic data and the sensory data "preference" of Red Globe grape.

main differences in the correlograms of the white varieties compared to the red one are in the region of $5000-7000 \mathrm{~cm}^{-1}$ (Fig. 6).

A tentative attribution of high correlation coefficients to specific molecular vibrations is not easy, anyway absorption bands around 6978, 5643 and $5614 \mathrm{~cm}^{-1}$ are usually related to sugars and organic acids [12]. The absorption bands at $6900 \mathrm{~cm}^{-1}$ are also related to a combination of stretch and deformation of $\mathrm{O}-\mathrm{H}$ group in glucose $[13,14]$. Therefore, it seems that the sugar and acidic composition of tested grapes influenced the preference of the panel especially for Red Globe. The differences in the correlation graphs show that the preference for the two white varieties and the red variety was not influenced by the same grape properties. Since one of the main differences among the three varieties is the colour it might be one or maybe the main responsible of difference in grape appreciation. Anyway, pigments are usually better detected with spectrophotometric techniques in the UV-Vis range, than with NIR spectroscopy. The lack of a strong difference between the two white varieties is surprising because these cultivars are different in terms of overall appearance and chemical composition. Probably the factors that mainly influenced tasters in the panel employed were mainly linked to texture related parameters which are linked to water content (positive correlation for lower and higher wave numbers, regions mainly linked to water signals). Further investigations to get a deeper understanding of the tasters preference and to confirm our findings are an ongoing.

\section{References}

[1] OIV (International Organisation of Vine and Wine). Resolution VITI 1/2008 Standard on Minimum Maturity Requirements for Table Grapes

[2] Food and Agriculture Organization of the United Nations (FAO). Codex Standard for Table Grapes (CODEX STAN 255-2007) Amended 2011

[3] European Union (EU). Commission Implementing Regulation (EU) No 543/2011 of 7 June 2011

[4] BIO Web of Conferences 9, 01028 (2017), 40th World Congress of Vine and Wine

[5] P. Cane, L'Enotecnico 26, 69 (1990)

[6] Association de coordination technique pour l'industrie agro-alimentaire (ACTIA) (2001). Sensory evaluation guide of good practice. Technical Report, Technical Coordination Association for the food industry, Paris, France

[7] V. Jayasena, I. Cameron, J. Food Qual. 31, 736 (2008)

[8] M. Le Moigne, C. Maury, D. Bertrand, F. Jourjon, J. Food Qual. Pref. 19, 220 (2008)

[9] P. Williams, K.H. Norris, Near Infrared Technology in the Agriculture and Food Industries 2nd ed. (St Paul: The American Association of Cereal Chemists, 2001)

[10] J.A. Guthrie, K.B. Walsh, D.J. Reid, C.J. Liebenberg, Aust. J. Agric. Res. 56, 405 (2005)

[11] L. Magwaza, U.L. Opara, H.H. Nieuwoudt, P.J.R. Cronje, W. Saeys, B.N. Nicolaï, Food Bioprocess Tech. 5, 425 (2011)

[12] F. Liu, Y. He, L. Wang, G. Sun, Food Bioprocess Tech. 4, 1331 (2011)

[13] P.A. Da Costa Filho, Anal. Chim. Acta 631, 206 (2009)

[14] E. Ferrari, G. Foca, M. Vignali, L. Tassi, A. Ulrici, Anal. Chim. Acta 701, 139 (2011) 